AntónIO Gonçalo InOCÊnCIO VILA Verde ${ }^{1}$ António COSTA Braga ${ }^{1}$ Daniela Reis Gonçalves ${ }^{1}$

Ana Rita Nunes Machado Morera Galvão' Sofia PINA RodRIGUes ${ }^{1}$ JOANA FILIPA FiguerREDO MACEDO ${ }^{2}$ MARIA do Céu Rodrigues ${ }^{1}$

Artigo Original

Palavras-chave

Artéria subclávia Aorta torácica

Diagnóstico pré-natal

Ultrassonografia

Primeiro trimestre da gravidez

Keywords

Subclavian artery Aorta, thoracic Prenatal diagnosis Ultrasonography Pregnancy trimester, first

\title{
Avaliação da artéria subclávia direita na ecografia do primeiro trimestre
}

\author{
Right subclavian artery evaluation during first trimester \\ ultrasound scan
}

\section{Resumo}

OBJETIVO: Avaliar a artéria subclávia direita durante a ecografia do primeiro trimestre, descrever a técnica para a sua avaliação e, em caso de identificação de artéria subclávia direita aberrante (ARSA), determinar sua associação com alterações cromossómicas e/ou malformações cardíacas e sua orientação. MÉTODOS: Estudo prospectivo que consistiu na avaliação da artéria subclávia direita no primeiro trimestre (comprimento crânio caudal entre 45 e 84 milímetros), em todas as gestações únicas, consecutivas, por um único examinador, ecógrafo Voluson E8 (GE Healthcare, Zipf, Áustria) com sonda transabdominal RAB 4-8-D, 2 a $8 \mathrm{MHz}$, por um período não superior a 2 minutos, numa população geral de baixo risco. Com a ajuda do power/color Doppler, a artéria subclávia direita foi classificada como normal ou aberrante. Foi utilizada análise de regressão estatística (IBM SPSS Statistics for Windows, versão 20.0) para estudar o grau de associação entre a fallha na avaliação/ classificação da artéria subclávia direita e o comprimento crânio-caudal fetal e o índice de massa corporal materno. RESULTADOS: A mediana da idade materna foi de 30 anos (variando entre 17 e 43 anos) e a mediana do tempo de gestação no momento da avaliação da artéria subclávia direita foi de 12 semanas de gestação (variando entre 11 e 13 semanas de gestação). A avaliação da artéria subclávia direita foi possível em 138/176 (78,4\%) dos casos. ARSA foi diagnosticada em um único caso $(0,7 \%)$. Esse feto com ARSA também apresentou um foco hiperecogênico no ventrículo cardíaco esquerdo. Foi realizada ecocardiografia fetal às 16 semanas de gestação, que confirmou o diagnóstico de ARSA e foco hiperecogênico. A amniocentese revelou cariótipo normal, 46, XX. CONCLUSÃO: É possível fazer o diagnóstico de ARSA na ecografia do primeiro trimestre. $\bigcirc$ nosso único caso de ARSA apresentou um cariótipo normal sem malformações cardíacas associadas.

\section{Abstract}

PURPOSE: To determine the feasibility of evaluation of the right subclavian artery during the first trimester ultrasound scan, as well as to describe the technique for its evaluation and, in case of aberrant right subclavian artery (ARSA) identification, to determine its association with chromosomal abnormalities and/or cardiac malformations and its management. METHODS: A prospective study for evaluation of the right subclavian artery during the first trimester ultrasound scan (crownto-rump length between 45 and $84 \mathrm{~mm}$ ), in all consecutive single pregnancies, by a single examiner, using a Voluson E8 system (GE Healthcare, Zipf, Austria) with a 2 to $8 \mathrm{MHz}$ RAB 4-8-D transabdominal probe, within a short period of time (less than 2 minutes), in a general low risk population. Color and/or power Doppler flow mapping was used to classify the right subclavian artery as normal or aberrant. Regression analysis with the IBM SPSS Statistics software for Windows, version 20.0 was used to determine the significance of the association between failure to examine/classity the right subclavian artery and both fetal crown-rump length and maternal body mass index. RESULTS: Median maternal age was 30 years (range: 17-43 years) and median gestational age at the time of evaluation of the right subclavian artery was 12 weeks (range: 1 1-13 weeks). The evaluation of the right subclavian artery was successful in 138/176 (78.4\%) of the cases. ARSA was diagnosed in a single case $(0.7 \%)$. This fetus with ARSA also presented a hyperechogenic focus on the left cardiac ventricle. Fetal echocardiography at 16 weeks of gestation was performed and confirmed ARSA and the hyperechogenic focus. Amniocentesis revealed a normal 46, XX karyotype. CONCLUSION: ARSA can be identified during a routine first trimester ultrasound scan. Our single ARSA case had a normal karyotype and no associated cardiac malformations.

Correspondêncio Goncalo Inocêncio Rua da Torrinha, 227 4050 Porto, Portuga

Recebido $22 / 01 / 2015$

Aceito com modificacọoes 06/05/2015
Serviço de Obstetrícia e Ginecologia do Centro Hospitalar do Porto, Centro Materno Infantil do Norte - Porto, Portugal.

'Centro Hospitalar do Porto, Centro Materno Infantil do Norte - Porto, Portugal.

${ }^{2}$ Unidade de Saúde Familiar de Esposende Norte - Esposende, Portugal.

Conflito de interesses: não há. 


\section{Introdução}

Geralmente, o arco aórtico dá origem a três vasos: o tronco braquiocefálico, a artéria carótida comum esquerda e a artéria subclávia esquerda. $\mathrm{O}$ tronco braquiocefálico, por sua vez, divide-se originando a artéria subclávia direita e a artéria carótida comum direita.

Raramente, durante a embriogénese e desenvolvimento do arco aórtico, pode ser criada uma variante do normal, na qual o arco aórtico, em vez de originar três vasos, dá origem a quatro vasos: artéria carótida comum direita, artéria carótida comum esquerda, artéria subclávia esquerda e artéria subclávia direita. Nesxe caso, a artéria subclávia direita origina-se diretamente da aorta, sendo designada por artéria subclávia direita aberrante (aberrant right subclavian artery - ARSA). O nome 'aberrante' deve-se ao fato de se originar anormalmente à esquerda da linha média, passando posteriormente ao esófago e traqueia, para irrigar o braço direito.

Apesar de, na grande maioria dos casos, a ARSA ser uma variante do normal, não havendo sintomatologia ou implicações cardiovasculares, estão descritos casos em que a ARSA pode causar sintomas como disfagia ou dispneia por compressão esofágica ou da traqueia, respectivamente ${ }^{1-3}$. Além disso, sua existência está associada a cromossomopatias, nomeadamente trissomia do 21, e/ou malformações cardíacas ${ }^{4}$. Vários estudos de diagnóstico pré-natal recentes estimam que a incidência de ARSA varie entre 7,8-37,5\% nos fetos com trissomia do 21 e $0,6-1,5 \%$ nos fetos cromossomicamente normais ${ }^{5-13}$.

O diagnóstico pós-natal de ARSA em ecocardiografia de rotina não é fácil, dificultando o cálculo da sua incidência, quer na população normal, quer nos indivíduos com cromossomopatia e/ou malformações cardíacas ${ }^{8}$. Em estudo de cadáver, ARSA foi identificada em 1,2\% dos indivíduos ${ }^{14}$.

O objetivo deste estudo foi determinar a exequibilidade da avaliação da artéria subclávia direita durante a ecografia do primeiro trimestre, bem como descrever a técnica para sua avaliação e, em caso de identificação de ARSA, determinar a sua associação com cromossomopatia e/ou malformações cardíacas e orientação.

\section{Métodos}

Estudo prospectivo de avaliação da artéria subclávia direita durante a ecografia do primeiro trimestre (feto vivo com comprimento crânio caudal - CCC - entre 45 e $84 \mathrm{~mm}$ ), em 176 gravidezes únicas consecutivas, durante um período de 5 meses (1 de fevereiro de
2014 a 30 de junho de 2014), numa população geral de baixo risco.

Todas as grávidas foram submetidas a ecografia de diagnóstico pré-natal pelo mesmo examinador (obstetra certificado para ecografia do primeiro trimestre pela Fetal Medicine Foundation) utilizando um equipamento Voluson E8 system (GE Healthcare, Zipf, Austria) com sonda transabdominal RAB 4-8-D (2 a $8 \mathrm{MHz}$ ) e submetidas a avaliação de risco combinado do primeiro trimestre para aneuploidias, tendo em conta: idade da grávida; translucência da nuca. Essa avaliação consta de duas dosagens no sangue materno colhidas no mesmo dia da ecografia do primeiro trimestre (subunidade beta da gonadotrofina coriónica humana - $\beta$-hCG - e proteína A plasmática associada à gravidez - PAPP-A).

Grávidas com factores de risco conhecidos para anomalias fetais (excluindo a idade materna) e/ou gravidezes múltiplas foram excluídas.

A avaliação da artéria subclávia direita foi tentada por um período não superior a dois minutos e classificada em: artéria subclávia direita normal; ARSA não possível de ser avaliada/classificada.

Os fetos diagnosticados com artéria subclávia direita normal foram submetidos novamente a avaliação da artéria subclávia direita durante a ecografia morfológica (que ocorreu entre a $20^{a}$ e a $22^{a}$ semana de gestação), pelo mesmo examinador do primeiro trimestre. Fetos com ARSA foram submetidos a ecocardiografia às 16 semanas de gestação por uma ecocardiografista experiente; fetos em que não foi possível classificar a artéria subclávia direita foram excluídos.

A técnica utilizada foi a seguinte: iniciação com um corte transversal do tórax fetal, com visualização das quatro câmaras cardíacas, com ampliação máxima; seguido de diminuição do ganho e colocação de uma box com color ou power Doppler; identificação com cor do preenchimento ventricular pós contracção auricular; seguido de deslocação da sonda (mantendo o corte transversal) no sentido do polo cefálico fetal, e identificação do sinal do $\mathrm{V}$, formado pelo arco aórtico e artéria pulmonar/ductus arteriosus; seguido de tentativa (mantendo o mesmo corte transversal) de colocação do $\mathrm{V}$ no sentido paralelo com a sonda, ou a coluna fetal na posição às 3 ou 9 horas; seguido de diminuição do pulse repetition frequency (PRF), idealmente para valores $\leq 1,8 \mathrm{kHz}$; por último, deslocação da sonda (mantendo sempre o corte transversal) novamente no sentido do polo cefálico, e identificação da artéria subclávia direita.

A artéria subclávia direita normal é identificada ao nível das clavículas, passando pela frente da traqueia, em direcção ao braço direito, com um trajecto tortuoso em forma de "S". 
ARSA é identificada ao nível do corte transversal/axial do arco aórtico (abaixo da posição da artéria subclávia direita normal ou esquerda), passando posteriormente à traqueia, em direcção ao braço direito, com um trajeto mais linear do que a artéria subclávia direita normal.

Como o estudo foi considerado de risco mínimo/nulo, num exame de diagnóstico de rotina, foi apenas obtido consentimento verbal das grávidas.

Foi utilizada análise de regressão estatística (IBM SPSS Statistics for Windows, versão 20.0) para estudar o grau de associação entre a falha na avaliação/classificação da artéria subclávia direita e o CCC fetal e índice de massa corporal (IMC) materno.

\section{Resultados}

Durante o período de cinco meses, a artéria subclávia direita foi avaliada em 176 gestações únicas no primeiro trimestre. A avaliação foi bem sucedida em 138/176 $(78,4 \%)$ casos. As principais razões que impossibilitaram a avaliação da artéria subclávia direita $(\mathrm{n}=38)$ na ecografia do primeiro trimestre foram: o elevado IMC $(r=0,5$; $\mathrm{p}<0,001)$ e o menor tempo de gestação/CCC $(r=-0.2$; $\mathrm{p}<0,001$ ) (Figura 1).
A mediana da idade das grávidas foi 30 anos (variando entre 17 e 43 anos). Dessas, 21,7\% tinha idade $\geq 35$ anos. A mediana do IMC foi $24,0 \mathrm{~kg} / \mathrm{m}^{2}$ (variando entre $18,4-39,7 \mathrm{~kg} / \mathrm{m}^{2}$ ). A mediana do tempo de gestação foi de 12 semanas (variando entre 11 e 13 semanas de gestação) e a mediana do CCC foi $67 \mathrm{~mm}$ (variando entre 46 e $84 \mathrm{~mm}$ ) (Figura 2).

Das 138 avaliações da artéria subclávia direita na ecografia do primeiro trimestre, ARSA foi diagnosticada em apenas 1 caso $(0,7 \%)$. O diagnóstico de ARSA não foi isolado pelo fato de se ter identificado um foco hiperecogênico no ventrículo esquerdo cardíaco. Não se visualizaram outras malformações, nomeadamente cardíacas, nem outros marcadores ecográficos de cromossomopatia (apresentava translucência da nuca normal, osso nasal presente, sem regurgitação da válvula tricúspide e ducto venoso normal). A grávida foi submetida a uma ecocardiografia fetal na $16^{a}$ semana, que confirmou o diagnóstico de ARSA (Figura 3) e a presença de foco hiperecogênico no ventrículo cardíaco esquerdo. Amniocentese foi realizada na $17^{\mathrm{a}}$ semana e revelou cariótipo normal, 46, XX.

Todos os fetos diagnosticados com artéria subclávia direita normal no primeiro trimestre $(\mathrm{n}=137)$ foram submetidos novamente a avaliação da artéria subclávia direita

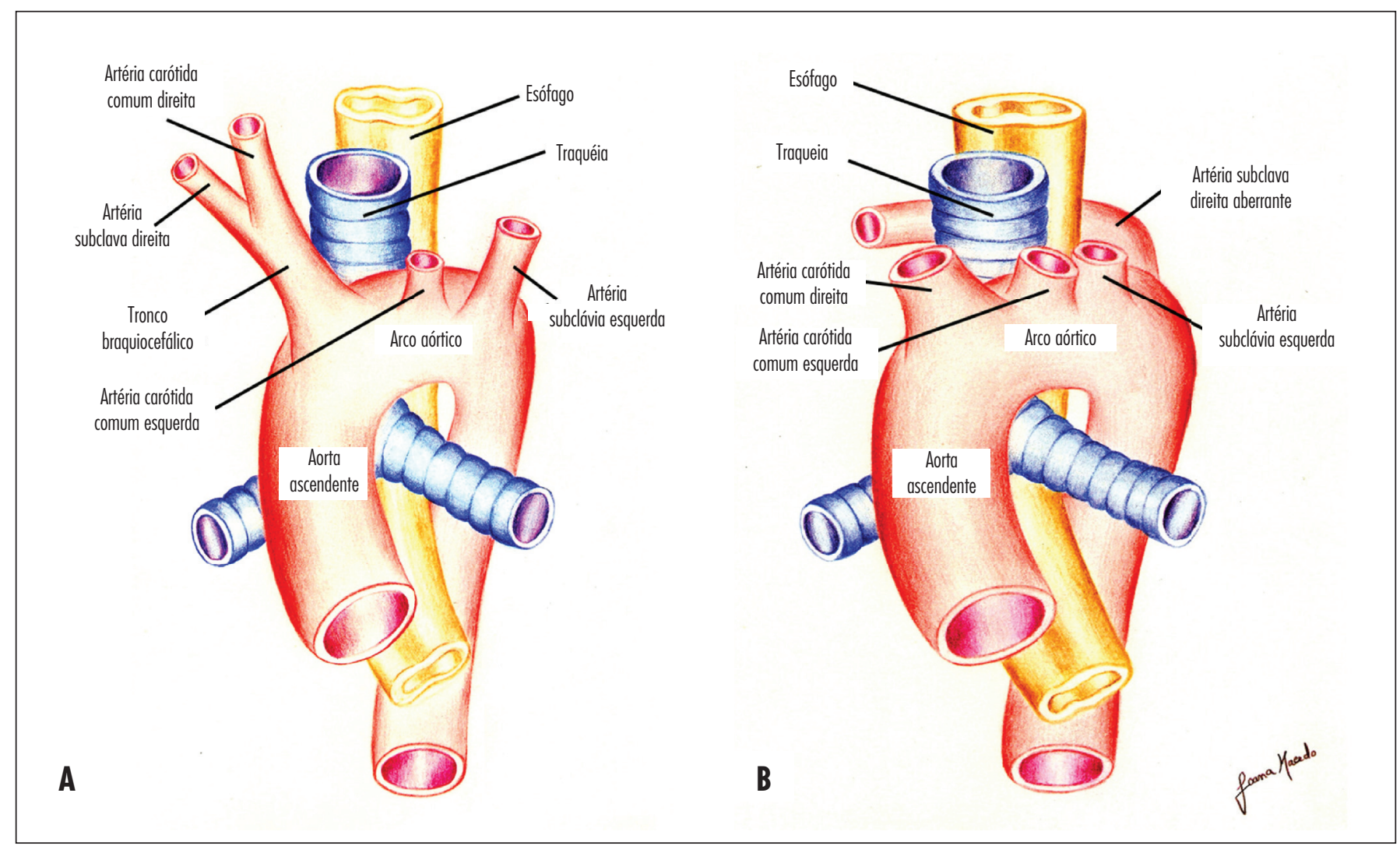

Figura 1. (A) Arco aórtico e artéria subclávia direita normal; (B) Arco aórtico com visualização da saída da artéria subclávia direita aberrante 


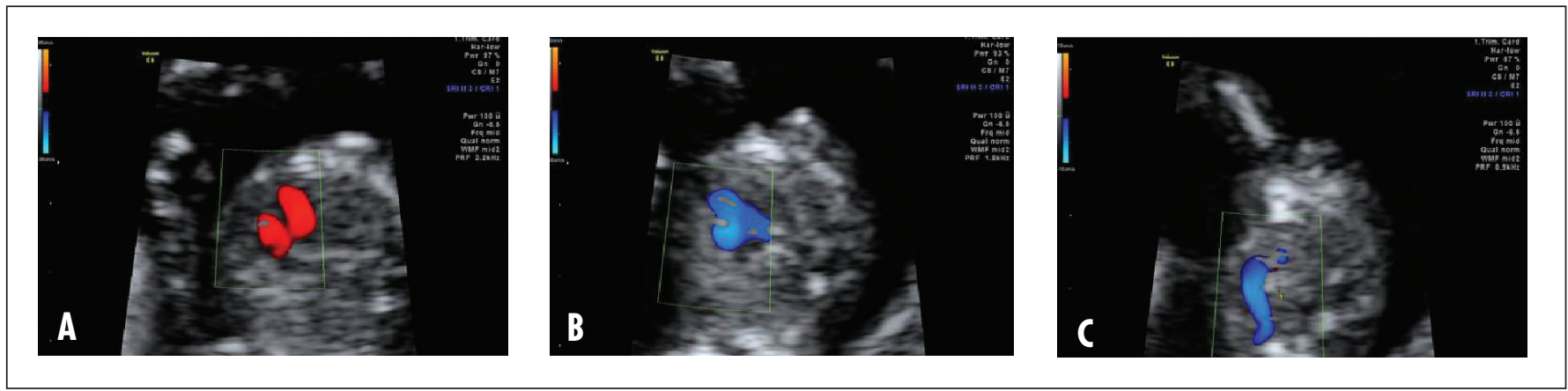

Figura 2. Feto com artéria subclávia direita normal às 12 semanas de gestação. (A) imagem ao nível das 4 câmaras cardíacas; (B) imagem ao nível do sinal do $\mathrm{V} ;(\mathrm{C})$ imagem ao nível da artéria subclávia direita normal

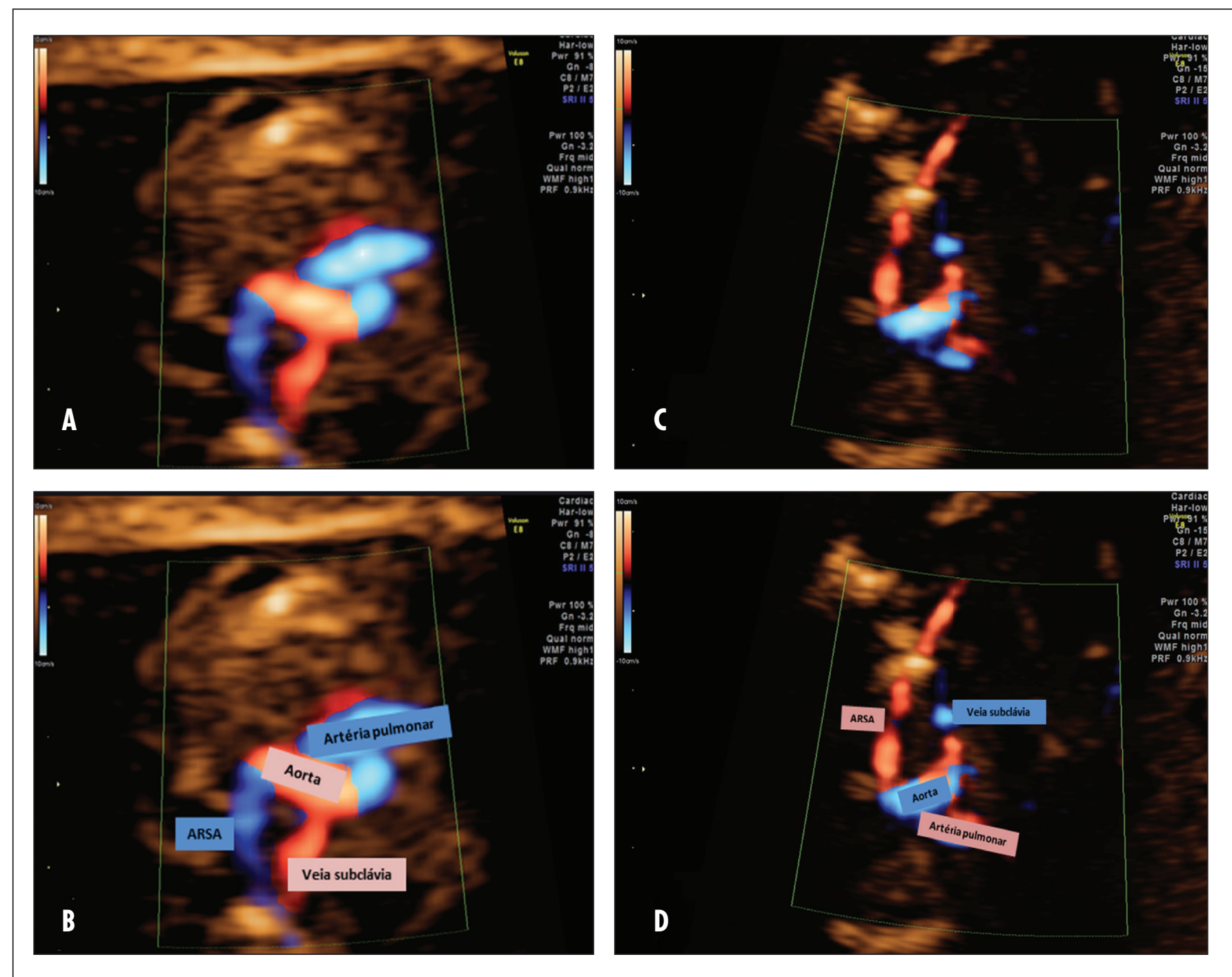

Figura 3. (A) Artéria subclávia direita aberrante diagnosticada em ecografia de rotina do primeiro trimestre, às 12 semanas de gestação; (B) legenda de $(A) ;(C)$ artéria subclávia direita aberrante confirmada no mesmo feto, em ecocardiografia fetal às 16 semanas de gestação; (D) legenda de (C)

durante a ecografia morfológica (mediana às 21 semanas de gestação), tendo sido possível classificar e havido concordância com o observado no primeiro trimestre em todos os casos.

Nas amostras incluídas não houve nenhum caso de risco combinado aumentado para cromossomopatias
(<1:250), diagnóstico de cromossomopatias (das submetidas ao exame invasivo; $n=14$ ), identificação de malformações fetais na ecografia morfológica do segundo trimestre (20-22 semanas de gestação), nem identificação de malformações cardíacas. 


\section{Discussão}

Relativo à técnica de avaliação da artéria subclávia direita: o aumento da imagem deverá ser obtido pela diminuição do ângulo de insonação e aumento máximo da profundidade, e não à custa do Pan Zoom - para que a frequência Hertz $(\mathrm{Hz})^{*}$, seja a máxima possível. Em outras palavras, quanto maior a frequência $\mathrm{Hz}$, mais imagens por segundo teremos, principalmente de um órgão como o coração, que no primeiro trimestre tem alta frequência/batimentos por minuto, para que a definição da imagem seja a melhor possível; não sendo obrigatório, por vezes escolhemos a cor do fundo como sépia pois pensamos ter maior contraste ajudando na melhor caracterização das estruturas cardíacas, bem como, na melhor identificação da traqueia, principalmente na ecografia do primeiro trimestre; pensamos ser igualmente importante a redução do ganho, antes da colocação do color ou power Doppler, assim como a diminuição do tamanho/ área da box do color ou power Doppler ao mínimo possível, pois permite melhorar a qualidade de recepção da cor; o objetivo de colocar o sinal do $\mathrm{V}$ em paralelo com a sonda, ou a coluna na posição às 3 ou 9 horas, é para que o ombro fetal (preferencialmente o direito) fique na direcção da sonda, para melhor captura da imagem da artéria subclávia direita e/ou esquerda simultaneamente; a redução do PRF antes da tentativa de visualizar a artéria subclávia direita é um passo importante, pois é um vaso com baixa velocidade e, portanto, pode não ser visualizado se o PRF não for reduzido.

O melhor plano para confirmação de ARSA, após a detecção no plano transversal/axial descrito, é o coronal ${ }^{12}$. $\mathrm{O}$ pequeno intervalo de tempo $(<2 \mathrm{~min})$ para avaliação da artéria subclávia direita foi escolhido por duas principais razões: tentar perceber se é possível obter mais um importante marcador ecográfico, num curto intervalo de tempo, e portanto não aumentando em demasia o tempo da ecografia do primeiro trimestre e por razões de segurança (pensa-se que o uso de Doppler num intervalo de tempo inferior a 5-10 min é seguro $)^{15,16}$.

Apesar de não ter sido um objetivo do nosso trabalho, surge a seguinte questão: perante o diagnóstico pré-natal de ARSA, e sabendo que por si só a ARSA aumenta o risco para trissomia do 21 cerca de 20-21,5 vezes ${ }^{8,17}$, será que deveremos oferecer um exame diagnóstico pré-natal invasivo, sempre que diagnosticamos uma ARSA? Na nossa opinião, a realização de exame diagnóstico pré-natal invasivo irá depender do risco para trissomia do 21 calculado pelo rastreio combinado do primeiro trimestre e/ou se existem outras malformações/marcadores associados. Uma opção será, após a

* 1 Hz É IGUAL A UM CICLO POR SEGUNDO. realização da ecografia morfológica, recalcular o risco para trissomia do 21 obtido no primeiro trimestre, e em função do resultado propor, ou não, exame de diagnóstico pré-natal invasivo. Para esse novo cálculo de risco poderá ser utilizada a tabela 11 do artigo "Meta-analysis of second-trimester markers for trisomy 21", publicado por Agathokleous et al. ${ }^{17}$, onde se pode observar que se ARSA for o único marcador isolado, há um aumento de 3,9 vezes, do risco para síndrome de Down, obtido no rastreio combinado do primeiro trimestre. Como no nosso caso o feto apresentava ARSA e um foco hiperecogênico cardíaco, o risco calculado do primeiro trimestre aumentou 28,7 vezes, tornando o rastreio positivo $(<1: 250)$, sendo proposta a realização de amniocentese que a grávida aceitou.

Aconselhamos também a ser sempre realizada ecocardiografia fetal em fetos com o diagnóstico de ARSA, pelo fato de que é um exame não invasivo e fetos com ARSA, mesmo que isolada, têm maior probabilidade de apresentarem malformações cardíacas congênitas. Nas situações em que houve necessidade de realização de cariótipo, há autores que defendem a exclusão da microdeleção do 22q11 (síndrome de DiGeorge) ${ }^{13}$.

Este estudo tem nomeadamente como limitações o fato das ecografias terem sido realizadas por um único examinador e o baixo número da amostra populacional com um único caso diagnosticado de ARSA. Um estudo mais amplo, com vários examinadores e/ou até multicêntrico, poderá relacionar o grau de concordância inter-observadores/ instituições, assim como avaliar o grau de correlação entre identificação de ARSA e sua associação com alterações cromossómicas e/ou malformações cardíacas e, portanto, determinar a importância da classificação da artéria subclávia direita na ecografia do primeiro trimestre.

Em conclusão, além de podermos afirmar que é possível a identificação de ARSA no primeiro trimestre, pensamos que a avaliação da artéria subclávia direita no feto é de grande importância pelas seguintes razões, de acordo com literatura recente $e^{4,8,18}$ : fetos com ARSA têm maior risco de apresentarem cariótipo anormal ${ }^{11,13,18}$; fetos com ARSA têm maior probabilidade de terem cardiopatias congênitas (mesmo os cromossomicamente normais) ${ }^{4,14,18}$; ARSA pode ser o único marcador isolado em fetos com trissomia $21^{4,8,18}$ — na nossa opinião, a curva de aprendizagem para avaliação da artéria subclávia direita para ecografistas com experiência em diagnóstico pré-natal é rápida e fácil (poderá ser realizado um estudo para contabilizar quantos exames ecográficos serão necessários até a aprendizagem da avaliação da artéria subclávia direita); é um marcador de avaliação exequível no primeiro trimestre e principalmente no $2^{\circ}$ trimestre (em $>95 \%$ dos casos é possível a sua avaliação $)^{8,18}$; não aumenta em demasia a duração do exame ecográfico (<2 minutos). 
1. Kent PD, Poterucha TH. Images in clinical medicine. Aberrant right subclavian artery and dysphagia lusoria. $\mathrm{N}$ Engl J Med. 2002;346(21): 1637 .

2. Yopp AC, Abrol S, Cunningham JN Jr, Lazzaro RS. Dysphagia lusoria and aberrant right subclavian artery. J Am Coll Surg. 2006;202(1):198.

3. Chaoui R, Heling KS, Sarioglu N, Schwabe M, Dankof A, Bollmann R. Aberrant right subclavian artery as a new cardiac sign in second- and third-trimester fetuses with Down syndrome. Am J Obstet Gynecol. 2005;192(1):257-63.

4. Gul A, Corbacioglu A, Bakirci IT, Ceylan Y. Associated anomalies and outcome of fetal aberrant right subclavian artery. Arch Gynecol Obstet. 2012;285(1):27-30.

5. Störk T, Gareis R, Krumbholz K, Eichstädt H. Aberrant right subclavian artery (arteria lusoria) as a rare cause of dysphagia and dyspnea in a 79-year old women with right mediastinal and retrotracheal mass, and co-existing coronary artery disease. VASA. 2001;30(3):225-8.

6. Zalel Y, Achiron R, Yagel S, Kivilevitch Z. Fetal aberrant right subclavian artery in normal and Down syndrome fetuses. Ultrasound Obstet Gynecol. 2008;31(1):25-9.

7. Borenstein M, Cavoretto P, Allan L, Huggon I, Nicolaides KH. Aberrant right subclavian artery at $11+0$ to $13+6$ weeks of gestation in chromosomally normal and abnormal fetuses. Ultrasound Obstet Gynecol. 2008;31(1):20-4.

8. Borenstein $M$, Minekawa $R$, Zidere $V$, Nicolaides $\mathrm{KH}$, Allan LD. Aberrant right subclavian artery at 16 to $23+6$ weeks of gestation: a marker for chromosomal abnormality. Ultrasound Obstet Gynecol. 2010;36(5):548-52.

9. Willruth AM, Dwinger N, Ritgen J, Stressig R, Gelpel A, Gembruch U, et al. Fetal aberrant right subclavian artery (ARSA): a potential new soft marker in the genetic scan? Ultraschall Med. 2012;33(7):E114-8.
10. Esmer AC, Gul A, Nehir A, Yuksel A, Dural O, Kalelioglu I, et al. Detection rate of trisomy 21 in fetuses with isolated and non-isolated aberrant right subclavian artery. Fetal Diagn Ther. 2013;34(3):140-5.

11. Fehmi Yazıcıoğlu H, Sevket $O$, Akın H, Aygün M, Özyurt $\mathrm{ON}$, Karahasanoğlu A. Aberrant right subclavian artery in Down syndrome fetuses. Prenat Diagn. 2013;33(3):209-13.

12. De León-Luis J, Gámez F, Bravo C, Tenías JM, Arias Á, Pérez $R$, et al. Second-trimester fetal aberrant right subclavian artery: original study, systematic review and meta-analysis of performance in detection of Down syndrome. Ultrasound Obstet Gynecol. 2014;44(2): 147-53.

13. Scala $C$, Leone Roberti Maggiore U, Candiani M, Venturini $\mathrm{PL}$, Ferrero S, Greco T, et al. Aberrant right subclavian artery in Down syndrome fetuses: a systematic review and meta-analysis. Ultrasound Obstet Gynecol. 2015 Jan 7. [Epub ahead of print].

14. Zapata H, Edwards JE, Titus JL. Aberrant right subclavian artery with left aortic arch: associated cardiac anomalies. Pediatr Cardiol. $1993 ; 14(3): 159-61$

15. Torloni MR, Vedmedovska $N$, Merialdi $M$, Betrán AP, Allen $T$, González R, et al. Safety of ultrasonography in pregnancy: WHO systematic review of the literature and meta-analysis. Ultrasound Obstet Gynecol. 2009;33(5):599-608.

16. Salvesen K, Lees C, Abramowicz J, Brezinka C, Ter Haar G, Marsál $K$, et al. ISUOG statement on the safe use of Doppler in the 11 to 13+6-week fetal ultrasound examination. Ultrasound Obstet Gynecol. $2011 ; 37(6): 628$.

17. Agathokleous M, Chaveeva P, Poon LC, Kosinski P, Nicolaides $\mathrm{KH}$. Meta-analysis of second-trimester markers for trisomy 21. Ultrasound Obstet Gynecol. 2013;41(3):247-61.

18. Rembouskos G, Passamonti U, De Robertis V, Tempesta A, Campobasso $G$, Volpe $G$, et al. Aberrant right subclavian artery (ARSA) in unselected population at first and second trimester ultrasonography. Prenat Diagn. 2012;32(10):968-75. 